\title{
Pattern of cardiac remodelling of the left ventricle in patients with essential hypertensive disease and concomitant type 2 diabetes mellitus
}

\author{
Authors: Aloysius Ikwuka and Natalia Virstyuk
}

\section{Introduction}

The aim of this research was to study the pattern of cardiac remodelling of the left ventricle in patients with essential hypertensive disease (EHD) and concomitant type 2 diabetes mellitus (DM2).

\section{Methods}

Sixty patients ( 36 female and 24 male) were examined for 3 months, their average age being $58.8( \pm 4.2)$ years and their age range from 40-70 years. Fifteen patients had EHD degree 1-2, stage II, treatment-compensated group I (GI); 15 patients had DM2 treatment-subcompensated (glycated haemoglobin $\left(\mathrm{HbA}_{1 \mathrm{c}}\right)$ from $7.0 \%$ to $11.0 \%$; group II (GII)); and 30 patients with EHD degree 1-2, stage II, treatment-compensated and concomitant DM2 treatment-subcompensated $\left(\mathrm{HbA}_{1 \mathrm{C}}\right.$ - from $7.0 \%$ to $11.0 \%$; group III (GIII)). The control group consisted of 20 healthy volunteers. Groups examined were randomised in age, sex, body mass index, duration of EHD and DM2. Research was conducted with strict adherence to Helsinki declarations concerning human research.

Echocardiography was used to determine the pattern of cardiac remodelling of the left ventricle according to the criteria of Ganau et al. ${ }^{1}$ Types of cardiac remodelling of the left ventricle:

> normal left ventricular geometry = normal index of myocardial mass of left ventricle (iMMLV) and relative thickness of left ventricular wall $(\mathrm{RTLVW})<0.44$ (iMMLV = MMLV/total body surface area (TBSA), where TBSA is calculated according to Mosteller's formula with the aid of a scientific calculator; RTLVW calculated according to formula)

> concentric left ventricular hypertrophy (concentric LVH) = presence of $L V H$ and RTLVW $\geq 0.45$

$>$ eccentric LVH = presence of LVH and RTLVW $<0.45$

$>$ concentric remodelling of left ventricle $=$ normal $\mathrm{MMLV}$ and $R T L V W \geq 0.45$

\section{Results and discussion}

According to the results of the echocardiography, normal left ventricular geometry was detected in three $(20.0 \%)$ GI patients $(p<0.05)$, in six $(40.0 \%)$ GII patients $(p<0.05)$ and in three $(10.0 \%)$ GIII patients ( $p<0.05)$; concentric LVH in $10(66.7 \%)$ GI, in eight $(53.3 \%)$ GII and in $14(46.7 \%)$ GIII patients ( $p<0.05)$; eccentric $\mathrm{LVH}$ in one $(6.7 \%)$ GII and in two $(6.7 \%)$ GIII patients $(p<0.05)$; and concentric remodelling of the left ventricle was detected in two (13.3\%) GI and $11(36.7 \%)$ GIII patients $(p<0.05)$.

\section{Conclusion}

Concentric LVH was detected most frequently and eccentric LVH was detected in rare cases $-<7.0 \%$ in patients with EHD and concomitant DM2.

\section{Reference}

1 Ganau A, Devereux RB, Roman MJ et al. Patterns of left ventricular hypertrophy and geometric remodeling in essential hypertension. J Am Coll Cardiol 1992;19:1550-8. 